\title{
Techniques for on-board vibrational passenger comfort monitoring in public transport
}

\author{
Ileana Bodini, Matteo Lancini, Simone Pasinetti, David Vetturi \\ University of Brescia - Department of Mechanical and Industrial Engineering, via Branze 38, 25123 Brescia, Italy
}

\begin{abstract}
Traffic calming devices on urban streets, such as elevated pedestrian crossings, speed bumps and roundabouts, are increasingly used, raising a real problem in relation to the on-board comfort that passengers perceive. To measure vibrational comfort related to traffic calming devices that passengers of the public transport perceive, an acquisition system called ASGCM (Autonomous System for Georeferenced Comfort Measurements) has been developed, taking as a reference the European regulations on rail transports. ASGCM permits to link each measurement of vibration, ground velocity and acceleration with geographical information resulting from a GPS. In this way a map of a comfort index, statistical surveys and correlation between on-board comfort and traffic calming, can be directly obtained by any Geographic Information System (GIS), able to query a centralized remote database, which was developed ad-hoc. A large number of experimental tests has been performed to define a vibrational comfort index and to collect a large dataset that allows statistically significant comparisons between different infrastructures and their characterization. The proposed technique can also be useful for diagnostics purposes, such as vehicle comparison and road maintenance state monitoring.
\end{abstract}

\section{Section: RESEARCH PAPER}

Keywords: Vibrational on-board comfort, geo-referenced comfort measurement, thematic maps

Citation: Ileana Bodini, Matteo Lancini, Simone Pasinetti, David Vetturi, Techniques for on-board vibrational passenger comfort monitoring in public transport, Acta IMEKO, vol. 3, no. 4, article 7, December 2014, identifier: IMEKO-ACTA-03 (2014)-04-07

Editor: Paolo Carbone, University of Perugia

Received October $11^{\text {th }}, 2013$; In final form January $14^{\text {th }}, 2014$; Published December 2014

Copyright: (C) 2014 IMEKO. This is an open-access article distributed under the terms of the Creative Commons Attribution 3.0 License, which permits unrestricted use, distribution, and reproduction in any medium, provided the original author and source are credited

Funding: (none reported)

Corresponding author: Ileana Bodini, e-mail: Ileana.bodini@ing.unibs.it

\section{INTRODUCTION}

The use and the development of collective transport systems are encouraged by public institutions in order to improve mobility and accessibility in urban areas. These aspects are also key elements in the development of "smart cities". Therefore, the increase of service quality and safety is fundamental in making public transport systems more attractive than private vehicles [1].

Service quality is usually evaluated in terms of accessibility to the transport system, timing flexibility and other properties of the transport network, while a comfort analysis is usually focused on the vehicle itself, even if its results depend also on roads and infrastructures.

Developments in laws [2] and some researches [3, 4, 5] have put in evidence two different aspects that could seem to be in contrast: one is the necessity to improve road safety, by introducing traffic calming measures, such as roundabouts, speed bumps, chicanes, elevated pedestrian crossings or different types of pavements; the second is the attention to the exposure of workers and passengers to vibration also on public transport vehicles, and its effect on transport quality. Until now many authors have been studying traffic calming devices and public transport passengers interaction $[6,7,8,9]$.

The purpose of this work is to give useful information to the designers of both vehicles and infrastructures; in particular this research is aimed at defining a technique to evaluate how traffic calming devices affect the acceleration that road public transport passengers perceive and to provide a low-cost device to easily measure and monitor vibration level on road public transport. Therefore, taking as a reference the European regulations on rail transports [10], the researchers propose the definition of a vibrational comfort index that allows to characterize and compare different road infrastructures and that could also be useful to compare behaviours of vehicles related to road infrastructure and to monitor vehicle maintenance state. 


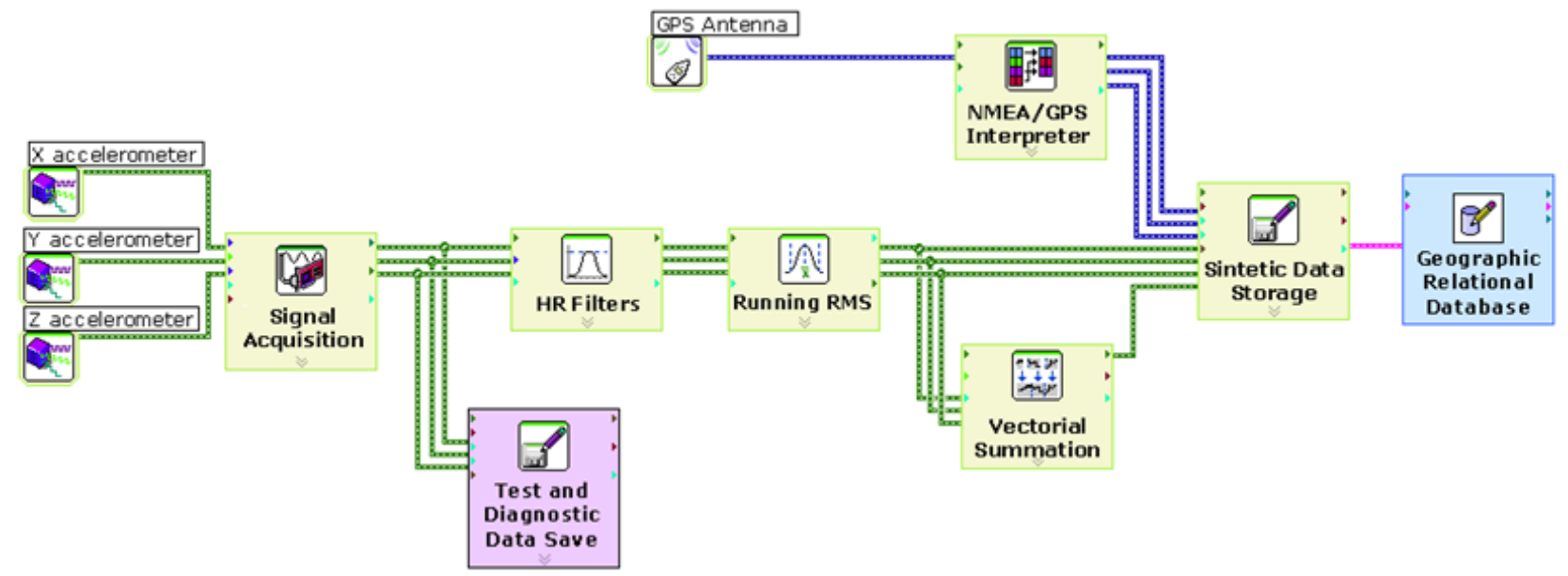

Figure 1. Flow chart of the automated acquisition procedure of ASGCM.

To calculate a comfort index and to correlate it with a given location, accelerations and GPS positions are needed. In Section 2 an ASGCM (Autonomous System for Geo-referenced Comfort Measurements) measurement system, that consists of the measurement chain and the processing system that allow to collect data and make them available, is described. In Section 3 the proposed comfort index is defined, and in Section 4 different types of data analysis are proposed. Finally, in Section 5 , the comfort index validation is discussed.

\section{MEASUREMENT AND PROCESSING SYSTEM}

To assess the on-board comfort that bus passengers perceive due to traffic calming devices, an ad-hoc measurement system, called ASGCM (Autonomous System for Geo-referenced Comfort Measurements) has been developed. This is autonomous for long periods of time and acquires, processes, and records data without requiring specialized staff [11]

To monitor on-board comfort, two quantities are needed: comfort level and geographical location. The measurement chain consists of a capacitive triaxial accelerometer mounted along the three principal orthogonal axes, a NI Compact-RIO, an analogue input module, supply and connection cables, a commercial GPS antenna, and an USB Flash memory. Both the accelerometer and the NI Compact-RIO are battery-powered.

The comfort level has been calculated taking into account both infrastructural effects, involving low frequencies, and vibration effects. These have been measured using a triaxial accelerometer, with an acquisition rate of $1000 \mathrm{~Hz}$ and a buffer of 1000 samples. In one second of acquisition the buffer is filled up and data are processed using a band-pass filter, from $0.5 \mathrm{~Hz}$ to $300 \mathrm{~Hz}$, designed according to the human response to vibration filters $[10,12]$ : acceleration magnitude is computed as a vector sum of the three accelerations acquired in orthogonal directions (the $\mathrm{x}$-axis corresponds to the road direction, the $\mathrm{y}$ axis is in the road plane and orthogonal to the x-axis, and the $z$ axis is perpendicular to the road plane) and an RMS acceleration value is calculated, considering a period of 5 seconds. This period of time represents a compromise between the acquisition of low frequency vibrations and a statistically relevant number of samples.

The time needed to fill the buffer is adequate to query the GPS: each second a standard NMEA (National Marine Electronics Association) RMC (Recommended Minimum sentence C) string is recorded [13]. This string contains information of position, velocity and time. Due to this procedure, illustrated in Figure 1, information of vibration, position and velocity related to the same second, are associated with a single geographical point.

\section{INDEX CHOICE}

The European regulation on rail transport [10] defines different types of comfort index; the one for standing passengers is called NVD. This has been adapted for road transport and has been calculated following equation (1),

$N V D=3 \cdot \sqrt{16 \cdot a_{x_{w}}^{2}+4 a_{y_{w}}^{2}+a_{z_{w}}^{2}}+5 \cdot a_{y_{w}}$

where $a_{x_{w}}$ is the RMS (Root Mean Square) weighed longitudinal acceleration, $a_{y_{w}}$ is the RMS weighed lateral acceleration and $a_{z_{w}}$ is the RMS weighed vertical acceleration. Weighing is performed following European regulations and considering the human response to vibration frequency filters $[10,12]$. The higher the NVD value the greater the on-board discomfort.

As stated before, each second, this comfort index is linked to vehicle velocity and GPS coordinates and the results are updated to a remote database which could then be queried, in order to obtain thematic maps and statistical analyses.

\section{ANALYSIS RESULTS}

\subsection{Analysis of manoeuvres and infrastructures}

Querying the developed database, kinematic parameters, such as speed and accelerations, related to vehicle vibrational comfort, can be analyzed. As a first result these parameters can be plotted as a function of time and position, as shown in Figure 2.

This type of analysis gives detailed information strictly related to every single passage of a chosen vehicle through a considered infrastructure, which can therefore be very accurately studied. However this kind of analysis is not very appropriate to monitor a complete public transport network or to evaluate an infrastructure design, because many external parameters such as driver, weather, bus crowding and traffic conditions can affect the measurement. These are important 

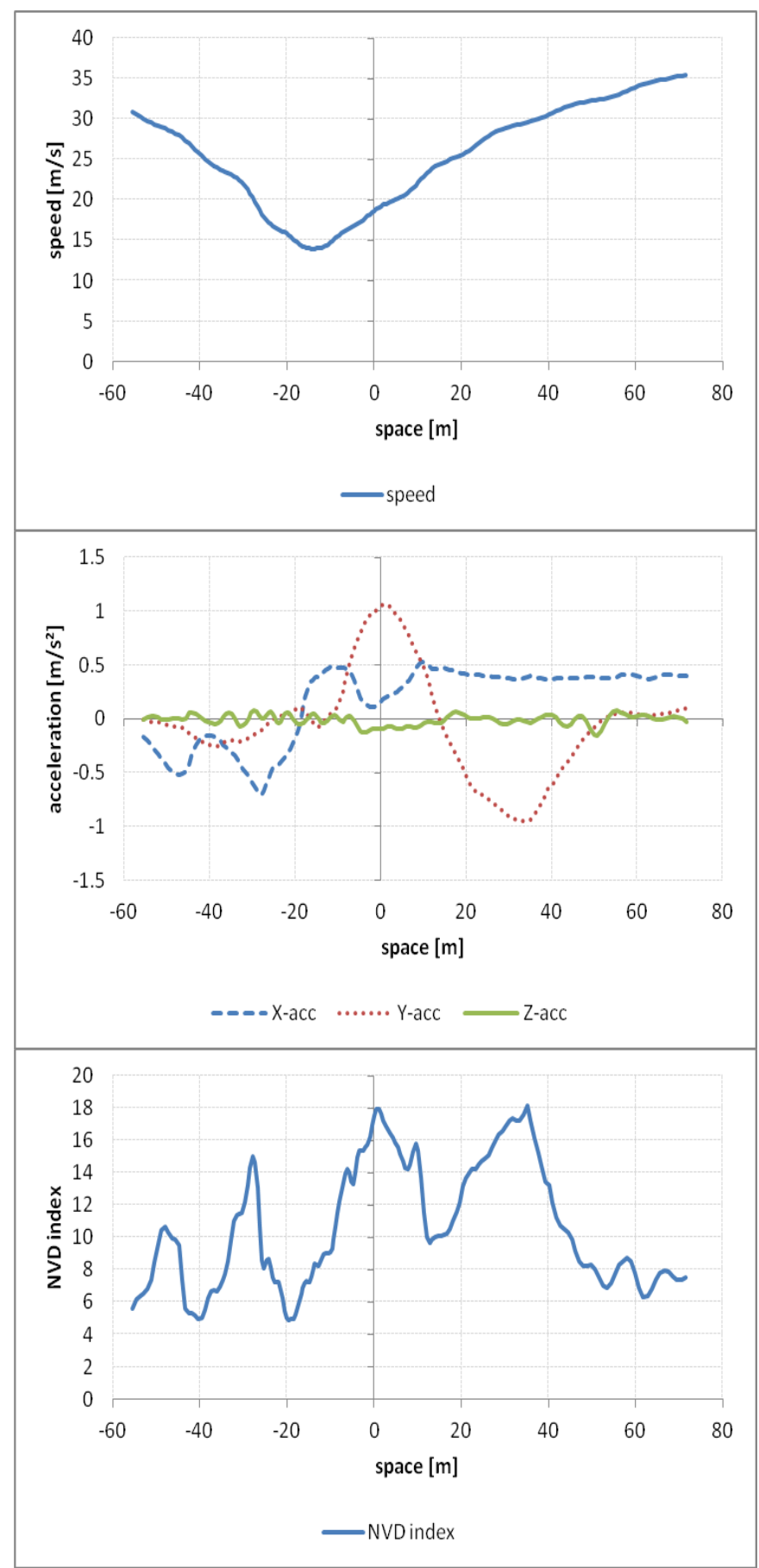

Figure 2. Kinematic parameters (speed and acceleration) and NVD index on the same roundabout.

factors that can be considered, for example, when discomfort causes have to be investigated.

\subsection{Geographical analysis}

To obtain less detailed but more significant information, the acquired data have to be aggregated. A first approach consists in a geographically-based aggregation obtained by dividing the urban map in a grid of a chosen step (for example $1 \mathrm{~m}$ ), and then associating to each cell the mean value of the parameter under study, in particular the NVD index. To take into account the geographical distribution of the data, the generic parameter is weighed using the inverse of the squared distance between the centre of the cell and the recorded position of each sample, following the widely accepted Inverse Distance Weighing

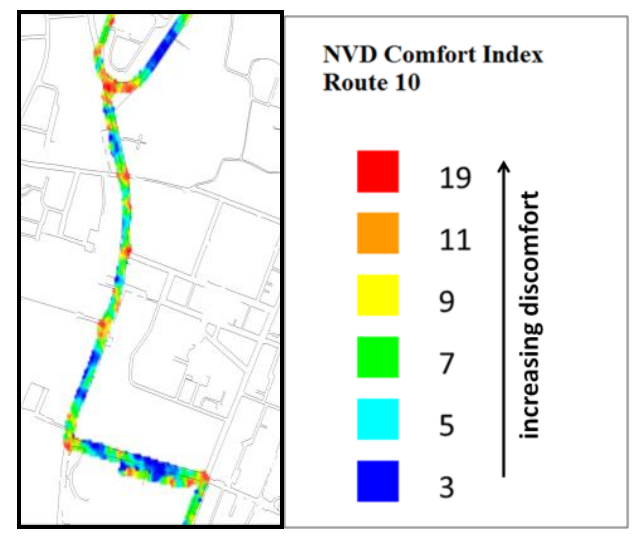

Figure 3. Thematic map of a portion of a bus route of the NVD comfort index, superimposed on the city cartography.

method. In this way a mean value of the chosen parameter has been calculated, taking into account both geographical distribution of the data and different runs of the vehicle, in different conditions, in the same geographical position.

Figure 3 shows a thematic map of the comfort index of a particular bus route as an average of a large number of acquisitions. In particular the bus route is superimposed on the city cartography: very uncomfortable spots (in red) as well as comfortable ones (in blue) are evidenced.

In general, thematic maps can be very useful to correlate a physical quantity, such as vehicle speed, acceleration vector, onboard comfort, with a given infrastructure, for example a roundabout, a speed bump or an elevated pedestrian crossing.
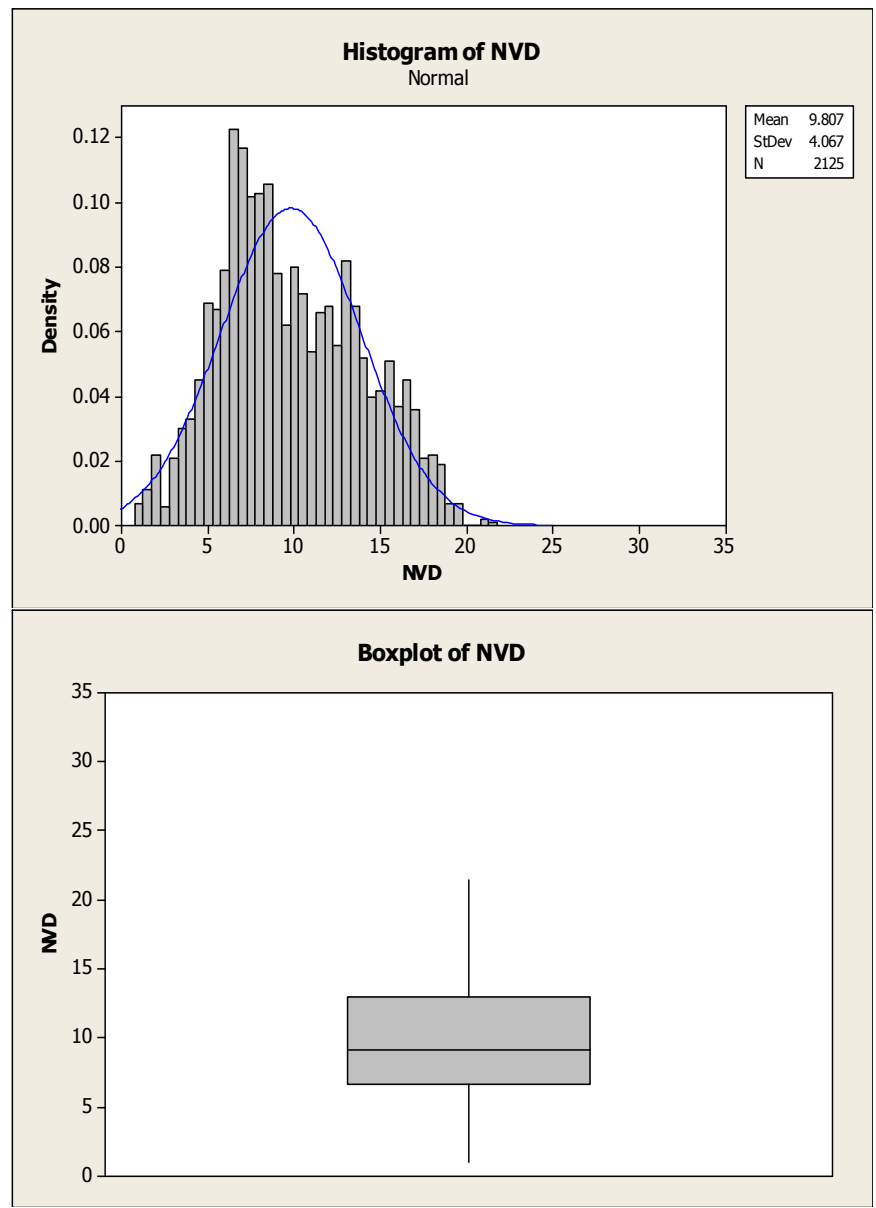

Figure 4. NVD comfort index statistical analysis. 


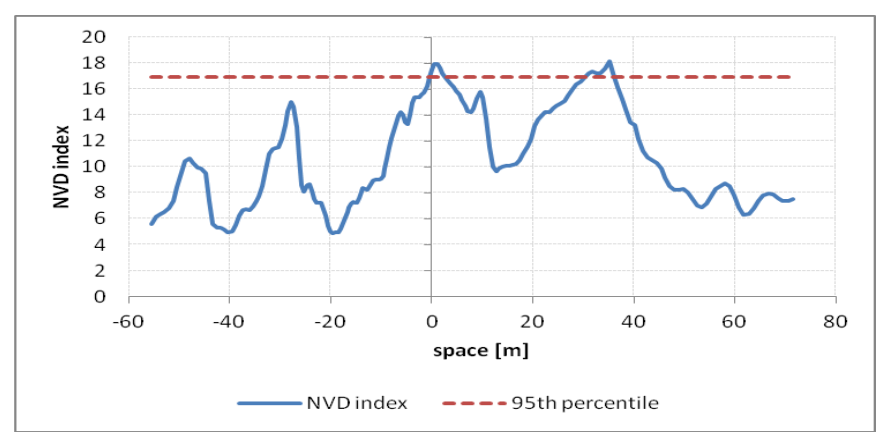

Figure 5. NVD comfort index $-95^{\text {th }}$ percentile.

However, using this kind of analysis, it is not possible to define different levels of index NVD related to real situations of comfort or discomfort, therefore thematic maps such as that shown in Figure 3 can be only used to define areas of relative discomfort with respect to the acquired data, without being able to point out which is actually the level of criticality itself.

\subsection{Statistical analysis}

A second approach to data aggregation consists in isolating some identified infrastructures or situations of interest, such as crossings, elevated pedestrian crossings, roundabouts, straight roads and considering the interesting physical quantities related to these infrastructures (such as vehicle speed, accelerations, on-board comfort) as stochastic variables over which a statistical analysis can be performed.

This allows to point out known situations of high comfort levels (straight roads with good pavement conditions) and low comfort levels (roundabouts, raised bumps) and to statistically investigate the corresponding NVD levels to develop a quantitative scale of the index of comfort.

The proposed method of analysis consists in selecting a path portion with fixed length, centred on the chosen infrastructure, and in assessing some important statistical parameters of the distribution of the variable of interest, in particular its Probability Distribution Function (PDF), its Cumulative Distribution Function (CDF), a box plot and the $95^{\text {th }}$ percentile of the distribution. This last parameter depends less on impulsive NVD index variations, not relevant from a statistical point of view, than the maximum NVD value, therefore, it has been chosen to summarize the complete distribution with a single number. A box plot is able, in particular, to synthetically indicate the trend of the probability distribution through the

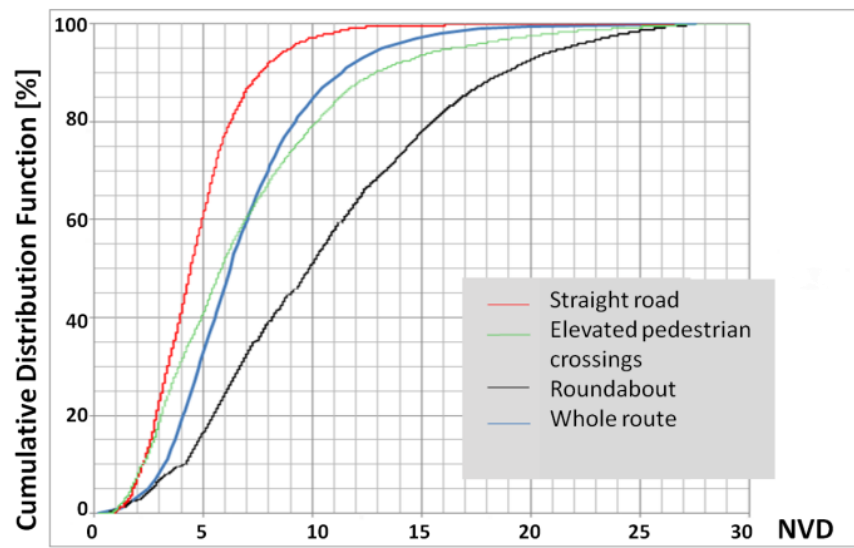

Figure 6. Comparison between cumulative distribution functions of the complete route for different infrastructures (straight roads, elevated pedestrian crossings and roundabouts).

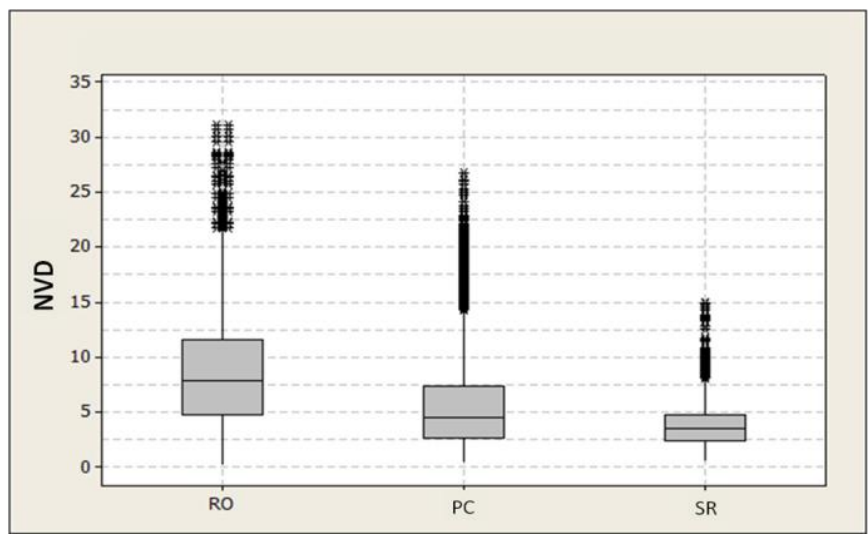

Figure 7. Comparison between box plots of different infrastructures (straight roads (SR), elevated pedestrian crossings (PC) and roundabouts (RO)) on the whole route.

\section{identification of its quartiles.}

As an example, referring to the roundabout proposed in Figure 2 and considering the NDV index as a stochastic variable, it is possible to study some statistical parameters, that give brief information about comfort (or discomfort) level related to the chosen infrastructure. Figure 4 shows the histogram of the distribution and a box plot of NVD indexes associated with the roundabout. A further example is given in Figure 5, which represents the NVD index of a single run as a function of position along the path (inside the inspection radius centred on the roundabout) and puts in evidence (dotted line) that the $95^{\text {th }}$ percentile of the NVD can be used to represent the worst on-board vibrational comfort condition, which occurs when the bus enters the selected infrastructure.

The complete statistical population includes all runs through an infrastructure (or through the whole route) of the same vehicle in different traffic, bus crowding, weather conditions and for different drivers. Each one of these parameters is a possible analysis criterion and a possible query for the database.

Following the described method, different statistical analyses have been performed; in particular, it is possible to represent cumulative distribution functions of the comfort index of a complete bus route or of a chosen type of infrastructure, such as roundabouts, elevated pedestrian crossings or straight roads. Figure 6 shows these cumulative distributions and allows to compare the comfort index of the whole route with that related to a particular type of infrastructure, giving brief information about on-board comfort of a specific bus route and allowing

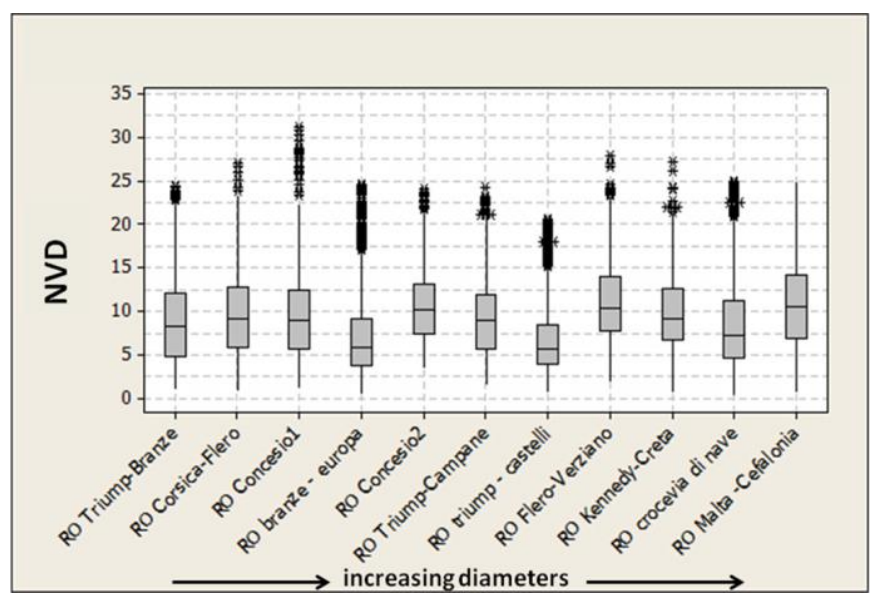

Figure 8. Comparison between box plots of different roundabouts. 


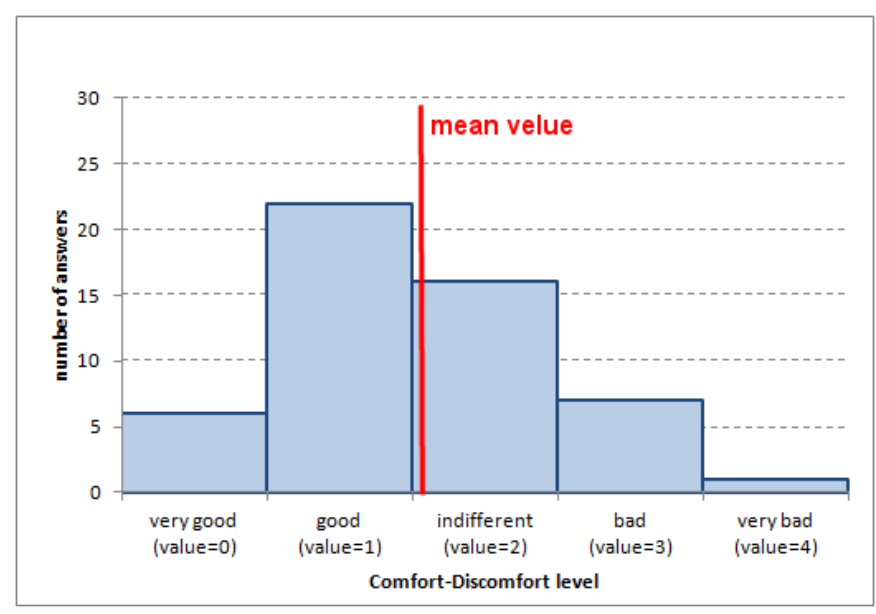

Figure 9. Relation between NVD index and panel evaluation.

for a comfort-based classification of specific traffic calming devices or road infrastructures. A comparison between comfort indexes related to different types of traffic calming devices and road infrastructures is also shown in Figure 7, using box plots as the statistical method.

Figure 8 shows that it is also possible to compare different traffic calming devices of the same type, such as roundabouts, and to study comfort index distribution of buses crossing such infrastructures. In particular, Figure 8 represents the comparison between different roundabouts of the same bus route and box plot are sorted according to diameter size; data were acquired using the same model of bus. Due to this kind of graph it is possible to deduce if roundabouts with different diameters present different levels of comfort. Another parameter that could be taken into account is the type of manoeuvre on roundabouts with the same geometry.

\section{INDEX VALIDATION}

To validate the proposed NVD Index, an analysis of correlation between instrumental comfort measurements and individual comfort perception has been performed. The individual perception of the comfort/discomfort level, on a selected bus route, has been evaluated by a test panel jury of more than 30 passengers who were asked repeatedly to express an evaluation of their perception in relation to different infrastructures (straight road, pedestrian crossing, roundabout, etc.), just few moments after their occurrence, for the whole duration of the chosen track.

The evaluation is given using a score that varies from 0 , when the situation is perceived as very comfortable, to 4 , when the situation is very uncomfortable. This ranking system is explained in advance to each subject to avoid misevaluation.

For all devices under investigation, mean value and standard deviation have been calculated. As an example, Figure 9 shows a distribution of jury responses on subjectively perceived comfort levels: in the graph each judgment (qualitative indication) is associated with a numerical value (quantitative indication) and the average value of judgment has also been reported.

To search for a significant correlation, the NVD index related to each studied infrastructure was considered a stochastic variable and the $95^{\text {th }}$ percentile of its distribution was chosen to synthesize the discomfort of the considered infrastructure. A linear regression was the result of a simple

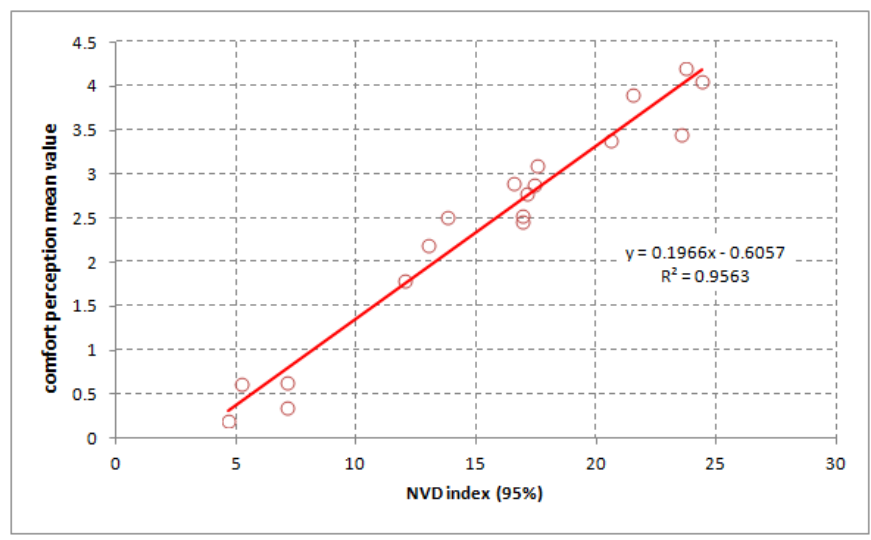

Figure 10. Linear relation between $95^{\text {th }}$ percentile of NVD index and panel evaluation.

least square approach [13], between this indicator and the average of the panel responses.

A linear relation has been found $(\mathrm{R}=96 \%)$ between the $95^{\text {th }}$ percentile of the NVD distributions and test panel opinions, associating the best comfort levels found (mean evaluation 0.2 - close to "very good" comfort) with an NVD level of 5 and the highest discomfort (mean evaluation 4 - "very bad") with an NVD level of 25. This relationship is shown in Figure 10 and could be used, as proposed by the authors, to define threshold values for comfort index.

\section{CONCLUSIONS}

A portable and autonomous measuring device for on-board comfort measurements on local transport buses has been set up, and specific software for retrieving from multiple devices, stocking and localizing data on the whole local transport network has been created. Automated tools for a geographical analysis of collected data with a standard GIS interface have also been developed and statistical analysis of the influence of specific traffic calming devices on on-board comfort was made feasible using the data collected.

A linear correlation between the results obtained by the measurement system and subjective perception of discomfort by a panel jury has been found, validating the authors' proposal of a modified NVD index for road vehicles for public transportation. In particular, the proposed analysis methodology allows, thanks to comfort thematic maps, to identify critical points, geographically localized, on a particular route, thus making possible a fast maintenance work if needed or pointing out comfort problems related to traffic calming devices or particular traffic and infrastructure conditions. Using the proposed index it is possible to compare different bus routes, different types of infrastructures, different types of bus travelling on the same route, different infrastructure of the same type that differs from geometry or for crossing manoeuvres.

Systematic acquisition of data regarding the whole transport network of a chosen area could allow to monitor the temporal evolution of vibrational comfort of the road network that is related to traffic conditions, road surface damages, presence of traffic calming devices. In this way, not only the general appreciation of the public transport system could be assessed, but also the temporal development of road conditions, as well as the interactions between subsequent traffic calming devices or road infrastructures. 


\section{ACKNOWLEDGEMENT}

Authors would like to thank Brescia Trasporti, and its bus drivers, for the support received, prof. G. Maternini for comments and insights and prof. F. Docchio for careful reading and discussion.

\section{REFERENCES}

[1] Tiboni M., Rossetti S., "Implementing a Road Safety Review Approach for Existing Bus Stops", WIT Transactions on the Built Environment, Vol. 130, pp. 699 - 709, WIT Press.

[2] European Parliament, "Directive 2002/44/EC of the European Parliament and of the Council of 25 June 2002 on the minimum health and safety requirements regarding the exposure of workers to the risks arising from physical agents (vibrations)", Official Journal, L 177 6.7.2002, p. 13.

[3] P. Eriksson, O. Friberg, Structural and multidisciplinary optimization 20 (2000) pp. 67-75.

[4] I.D. Jacobson, R.W. Barber, R.D. Pepler, L.L. Vallerie, Transportation Research Record 646 (1997) pp. 1-6.

[5] W.H. Park, J.C. Wambold, Transportation Research Record 584 (1976), pp. 55-63.
[6] G. Maternini, Silvia Foini, Tecniche di moderazione del traffico, Egaf edizioni, Forlì, 2010, ISBN 978-88-8482-364-9

[7] P. Jönsson, Ö. Johansson, Journal of Sound and Vibration 282 (2005), pp. 1043-1064.

[8] S. Grudemo, A. Ihs, M. Wiklund, "The influence of road surface condition on driving comfort" in VTI meddelande, 2004, vol. 957.

[9] K. Ahlin, N.O.J. Granlund, International Journal of Pavement Engineering (2002), pp. 207-216.

[10] .UNI EN 12299, "Railway applications, Ride comfort for passengers, Measurement and evaluation" (2009).

[11] Vettui D., Maternini G., Lancini M., Bodini I., "On-board comfort measuerement system development for buses", Proc. of the Seventh International Conference on Informatics and Urban and Regional Planning INPUT 2012, May 10-12, 2012, Cagliari, Italy, ISBN: 9788856875973, pp 1277-1286ISO 8041, "Human response to vibration - measuring instrumentation" (2005).

[12] SiRF, NMEA Reference Manual, SiRF Technology, Inc., California (2007).

[13] G.B. Rossi, B. Berglund, "Measurement related to human perception and interpretation - State of the art and challenges", XIX IMEKO World Congress Fundamental and Applied Metrology, Sept 6-11, 2009, Lisbon. 